\title{
Letter to editor: response to comment on risk of perioperative bleeding complications in rhegmatogenous retinal detachment surgery: a retrospective single-center study
}

\author{
Sebastian Bemme ${ }^{1}$ [D $\cdot$ Nicolas Feltgen ${ }^{1}$ \\ Received: 17 April 2020 / Revised: 17 April 2020 / Accepted: 25 April 2020 / Published online: 9 May 2020 \\ (C) Springer-Verlag GmbH Germany, part of Springer Nature 2020
}

Dear Editor,

We thank Kishore et al. for their interesting and constructive comment on our paper, which examined the rate of mild and severe bleeding complications after rhegmatogenous retinal detachment (RRD) surgery.

The rates of neither all nor severe bleedings in the phenprocoumon subgroup differed significantly from the acetylsalicylic acetate (ASA) subgroup or patients without anticoagulation. Indeed, our data allows no conclusions to be made about the perioperative bleeding risk in RRD surgery under anticoagulation with vitamin $\mathrm{k}$ antagonists (VKA) and an international normalized ratio within the therapeutic range or under anticoagulation with newer oral anticoagulants. Although they are already stated in the original article, we are grateful to Kishore et al. for highlighting these two aspects, as it is essential that our trial not be misinterpreted.

Regarding anticoagulation with ASA, 119 of the 140 patients of our ASA subgroup continued ASA during the 3 days before surgery, and most of them did not stop until their day of surgery. Having excluded the 21 patients stopping ASA $\geq$ 3 days before surgery still yielded no significantly different bleeding rates between ASA and control patients undergoing all types of surgery, nor with each type of surgery analyzed separately.

Nevertheless, we agree with the authors that the decision to stop or continue anticoagulation should be made for every patient individually. Online calculators such as the HASBLED Score may help surgeons to estimate the general bleeding risk to support their decision-making ability regarding anticoagulation. However, the bleeding risk also depends on surgical techniques, as our trial comparing buckling with and without subretinal drainage showed. A general bleeding score may not necessarily represent the actual bleeding risk in conjunction with a specific surgical intervention. Therefore, further studies are warranted to investigate the risk of bleeding during vitreoretinal surgery, especially in an emergency like RRD. A stricter categorization of bleeding complications like that the BARC criteria recommend might be helpful, but only adequately practicable for assessing the bleeding extent by the surgeon immediately after the operation or within the shortterm postoperative period, which underlines the necessity for prospectively designed trials on this issue.

Sebastian Bemme

sebastian.bemme@med.uni-goettingen.de

1 Department of Ophthalmology, University Medical Center Goettingen, Goettingen, Germany 